\title{
y-scaling in Quasielastic Electron Scattering from Nuclei
}

\author{
K. S. Kim ${ }^{1)}$ and L. E. Wright ${ }^{2)}$ \\ 1) School of Liberal Arts and Science, Korea Aerospace University, Koyang 200-1, Korea \\ 2)Institute of Nuclear and Particle Physics, Ohio University, Athens, OH 45701
}

A relativistic single particle model is used to calculate the inclusive $\left(e, e^{\prime}\right)$ reaction from $A=12,40,56,197$, and 208 nuclei in the quasielastic region. We have shown that this model provides a very good description of the available experimental cross sections when they are dominated by the quasielastic process. In this paper we use this model to investigate the dependence of $y$-scaling on electron kinematics, particularly the electron scattering angle, for a range of squared four momentum transfer $0.20-0.80(\mathrm{GeV} / \mathrm{c})^{2}$. In this kinematic domain, Coulomb distortion of the electron does not significantly affect scaling, but final state interactions of the knocked out nucleon do affect scaling particularly when the nucleons have lower energies. In general, we find that scaling works for this reaction, but at lower values of the four momentum transfer, the scaling function does have some dependence on the electron scattering angle. We also consider a modification of y-scaling to include small binding energy effects as a function of $\mathrm{Z}$ and $\mathrm{A}$ and show that there is some improvement in scaling.

PACS numbers: 25.30. Fj

Medium and high energy electron scattering has long been acknowledged as a useful tool to study nuclear structure and nuclear properties, especially in the quasielastic region where the process of knocking out nucleons is dominant. Many experiments [1, 2, 3], 4] have been performed on medium and heavy nuclei at incident electron energies less than $1 \mathrm{GeV}$ where contributions of inelastic processes can be avoided. There are also a number of theoretical works [5, 6, 7, 8, 9, 10, 11, 12] which have been compared to the measured responses.

As we have noted before [10, 11, 12], the Fermi gas model in the impulse approximation roughly describes the inclusive $\left(e, e^{\prime}\right)$ cross sections, but fails to provide a good description of the structure functions. A good theoretical description of quasielastic scattering requires two ingredients before one can compare experimental $\left(e, e^{\prime}\right)$ data from light to heavy nuclei 
to theory. One of them is a model for the nuclear transition current and the other is some provision for the inclusion of electron Coulomb distortion effects for medium and heavy nuclei. In the early 1990's, the Ohio University group [5, 13, 14] treated the electron Coulomb distortion exactly for the reactions $\left(e, e^{\prime}\right)$ and $\left(e, e^{\prime} p\right)$ in the quasielastic region using partial wave expansions of the electron wave functions in the distorted wave Born approximation (DWBA). The Madrid group [15] subsequently reported a similar analysis for the exclusive $\left(e, e^{\prime} p\right)$ reaction. However, the DWBA calculations do not allow a separation of the cross section into a longitudinal part and a transverse part and are numerically challenging, and computational time increases rapidly with higher incident electron energies. In order to avoid these difficulties, Kim and Wright [10, 11, 16] developed an approximate treatment of the electron Coulomb distortion which does allow the separation of the cross section into a longitudinal part and a transverse part.

In addition, we found a model which provides a very good description of quasielastic scattering processes from nuclei for both the inclusive and exclusive cases. It is a relativistic single particle model which requires the wave functions of bound and continuum nucleons and a transition current operator. The bound state wave functions are obtained from solving the Dirac equation in the presence of the strong vector and scalar potentials[17] and for the inclusive $\left(e, e^{\prime}\right)$ reaction where the knocked out nucleons are not observed, the continuum wave functions are solutions to a real potential so as not to lose any flux. At low energies, this potential is just the same as the bound nucleon potential and thereby guarantees current conservation and gauge invariance. However, it is known from elastic proton scattering that the continuum potential becomes weaker with increasing proton energy. Therefore, for higher energy processes we use a nucleon potential whose strength has been fitted to proton elastic scattering [18, 19]. In a recent paper [20], we found excellent agreement between our model with the higher energy experimental data from SLAC [21] for the quasielastic $\left(e, e^{\prime}\right)$ scattering on ${ }^{12} \mathrm{C},{ }^{56} \mathrm{Fe}$, and ${ }^{197} \mathrm{Au}$ at the squared four momentum transfer of approximately $0.20-0.30(\mathrm{GeV} / \mathrm{c})^{2}$ by using the energy-dependent real potentials (which are weaker than the bound state potentials) for the outgoing nucleons. Note that these calculations do not conserve nucleon transition current, but we calculate all four components of the transition current in order to minimize errors due to lack of current conservation. Note that the Madrid group [15] has used a very similar relativistic model for $\left(e, e^{\prime} p\right)$ reactions. The excellent agreement of this model with experimental data allow us to use it as a tool for investigating 
$y$-scaling of the quasielastic components of the inclusive $\left(e, e^{\prime}\right)$ even in kinematic regions where inelastic contributions have large contributions.

Since the pioneering work by West [22], there have been many experimental [4, 23] and theoretical [24, 25, 26, 27, 28, 29] investigations of $y$-scaling from nuclei. In this paper we propose to use our relativistic mean field single particle model with the inclusion of Coulomb corrections to investigate the approach to $y$-scaling at intermediate values of the four momentum transfer. Using $y$-scaling, the measured cross section for the inclusive $\left(e, e^{\prime}\right)$ reaction can be written as a product of the electron-nucleon cross section times a function $F$ which is related to the momentum distribution of nucleons in the nucleus and is a function of momentum transfer $q$ and energy transfer $\omega$. For the case of large momentum transfer, the function $F$ should depend only on a single variable $y$ which is a function of $\omega$ and $q$ [26]. Scaling is expected to be valid for the very large momentum transfer region, but it may be broken by final state interactions in the quasielastic region and/or electron Coulomb distortion effects. A SLAC experiment [21] was performed at the squared four momentum transfer $Q^{2}$ of $0.23-2.52(\mathrm{GeV} / \mathrm{c})^{2}$ and the data at $y<0$ exhibited scaling at large $Q^{2}$. Recently, there have been additional experimental data from JLAB [30] at $4.045 \mathrm{GeV}$, but scaling of the quasielastic process cannot be demonstrated since the energy transfer is sufficiently high that pion production is a significant contribution to the cross section. Note that both of these experiments have been carried out at relatively forward electron scattering angles. Furthermore, we cannot compare our model predictions to these data since we have not yet included inelastic processes (meson production, etc.) in our model. However, as noted above we can use our model to investigate the scaling of the quasielastic contributions to the cross section.

Using non-relativistic models, the authors in Ref. [27] analyzed $y$-scaling of the quasielastic electron scattering in few-body system, complex nuclei, and nuclear matter. Within the framework of the plane wave impulse approximation (PWIA), they investigated the effects of the final state interaction, the binding correction, and the nucleon-nucleon correlations. They pointed out that the relation between the scaling function and the momentum distribution does not exist at finite momentum transfer because of the final state interaction and the binding correction. More recently, the Madrid group in collaboration with Donnelly [29, 31, 32] have investigated scaling using a semi-relativistic model and note that the strong scalar and vector potentials in the final state of relativistic models result in a breakdown of 
scaling and result in different scaling functions for longitudinal and transverse responses.

In this paper, we initially calculate $y$-scaling at the squared four momentum $Q^{2}$ of approximately $0.2-0.3(\mathrm{GeV} / \mathrm{c})^{2}$ comparing with the experimental data measured at SLAC [21] for ${ }^{12} \mathrm{C},{ }^{56} \mathrm{Fe}$, and ${ }^{197} \mathrm{Au}$, and Bates [3] for ${ }^{40} \mathrm{Ca}$, where the quasielastic contribution is kinematically isolated from pion production. We then investigate the effects of final state interactions in our relativistic model and electron Coulomb distortion on scaling. Since we are considering cases with large outgoing nucleon energies, we do use an energy dependent final state interaction. Finally, we introduce a new $y$-scaling variable in order to solve the non-scaling problem in the presence of the final state interaction from different target nuclei in the same four momentum transfer range.

In the plane wave Born approximation (PWBA), where the electron wave functions are described by the Dirac plane waves, the cross section for the inclusive quasielastic $\left(e, e^{\prime}\right)$ reaction is written as

$$
\frac{d^{2} \sigma}{d \omega d \Omega_{e}}=\sigma_{M}\left\{\frac{q_{\mu}^{4}}{q^{4}} S_{L}(q, \omega)+\left(\tan ^{2} \frac{\theta_{e}}{2}-\frac{q_{\mu}^{2}}{2 q^{2}}\right) S_{T}(q, \omega)\right\},
$$

where $q_{\mu}{ }^{2}=\omega^{2}-\mathbf{q}^{2}=-Q^{2}$ is the squared four momentum transfer, $\sigma_{M}=$ $(\alpha / 2 E)^{2}\left[\cos ^{2}\left(\theta_{e} / 2\right) / \sin ^{4}\left(\theta_{e} / 2\right)\right]$ is the Mott cross section, and the longitudinal and transverse structure functions which depend on the three momentum transfer $q$ and the energy transfer $\omega$ are $S_{L}$ and $S_{T}$. Explicitly, the structure functions for a given bound state with angular momentum $j_{b}$ are given by

$$
\begin{aligned}
& S_{L}(q, \omega)=\sum_{\mu_{b} s_{p}} \frac{\rho_{p}}{2\left(2 j_{b}+1\right)} \int\left|N_{0}\right|^{2} d \Omega_{p} \\
& S_{T}(q, \omega)=\sum_{\mu_{b} s_{p}} \frac{\rho_{p}}{2\left(2 j_{b}+1\right)} \int\left(\left|N_{x}\right|^{2}+\left|N_{y}\right|^{2}\right) d \Omega_{p}
\end{aligned}
$$

with the outgoing nucleon density of states $\rho_{p}=\frac{p E_{p}}{(2 \pi)^{3}}$. The $\hat{\mathbf{z}}$-axis is taken to be along the momentum transfer $\mathbf{q}$ and the z-components of the angular momentum of the bound and continuum state nucleons are $\mu_{b}$ and $s_{p}$, respectively. The Fourier transform of the nuclear current $J^{\mu}(\mathbf{r})$ is simply given by

$$
N^{\mu}=\int J^{\mu}(\mathbf{r}) e^{i \mathbf{q} \cdot \mathbf{r}} d^{3} r
$$

where $J^{\mu}(\mathbf{r})$ denotes the nucleon transition current. The continuity equation could be used to eliminate the $z$-component $\left(N_{z}\right)$ via the equation $N_{z}=-\frac{\omega}{q} N_{0}$ if the current is conserved, but 
since we use an energy dependent final state interaction [20] we violate current conservation and to minimize errors calculate $N_{z}$ directly. The nucleon transition current in the relativistic single particle model is given by

$$
J^{\mu}(\mathbf{r})=e \bar{\psi}_{p}(\mathbf{r}) \hat{\mathbf{J}}^{\mu} \psi_{b}(\mathbf{r})
$$

where $\hat{\mathbf{J}}^{\mu}$ is a free nucleon current operator, and $\psi_{p}$ and $\psi_{b}$ are the wave functions of the knocked out nucleon and the bound state, respectively. For a free nucleon, the operator comprises the Dirac contribution and the contribution of an anomalous magnetic moment $\mu_{T}$ given by $\hat{\mathbf{J}}^{\mu}=F_{1}\left(q_{\mu}^{2}\right) \gamma^{\mu}+F_{2}\left(q_{\mu}^{2}\right) \frac{i \mu_{T}}{2 M} \sigma^{\mu \nu} q_{\nu}$. The form factors $F_{1}$ and $F_{2}$ are related to the electric and magnetic Sachs form factors given by $G_{E}=F_{1}+\frac{\mu_{T} q_{\mu}^{2}}{4 M^{2}} F_{2}$ and $G_{M}=F_{1}+\mu_{T} F_{2}$ which are assumed to take the following standard form:

$$
G_{E}=\frac{1}{\left(1-\frac{q_{\mu}^{2}}{\Lambda^{2}}\right)^{2}}=\frac{G_{M}}{\left(\mu_{T}+1\right)}
$$

where the standard value for $\Lambda^{2}$ is $0.71(\mathrm{GeV} / \mathrm{c})^{2}$.

The $y$-scaling function is defined as the ratio of the measured cross section to the off-shell electron-nucleon cross section as follows:

$$
F(y)=\frac{d^{2} \sigma}{d \omega d \Omega_{e}}\left(Z \sigma_{e p}+N \sigma_{e n}\right)^{-1} \frac{q}{\left[M^{2}+(y+q)^{2}\right]^{1 / 2}},
$$

where $\sigma_{e p}\left(\sigma_{e n}\right)$ denotes the off-shell electron-proton(neutron) cross section $\sigma_{c c 1}$ defined by Ref. [33]. In Eq. (7), $Z$ and $N$ are the number of protons and neutrons, and $M$ is the mass of nucleon. The scaling variable $y[34]$ is given by

$$
\omega+M_{A}=\left(M^{2}+q^{2}+y^{2}+2 y q\right)^{1 / 2}+\left(M_{A-1}^{2}+y^{2}\right)^{1 / 2},
$$

where $M_{A}$ is the mass of the target nucleus and $M_{A-1}$ is the mass of the ground state of the $A-1$ nucleus. The point $y=0$ corresponds approximately to the peak of the quasielastic scattering and $y<0(y>0)$ corresponds to the small (large) $\omega$ region.

In Fig. 1, we calculate the $y$-scaling functions for ${ }^{12} \mathrm{C},{ }^{40} \mathrm{Ca},{ }^{56} \mathrm{Fe},{ }^{197} \mathrm{Au}$, and ${ }^{208} \mathrm{~Pb}$ by neglecting both the final state interaction of the exiting nucleons and the electron Coulomb distortion. This is normally referred to as the plane wave impulse approximation (PWIA). The squared four momentum transfer is approximately $0.2(\mathrm{GeV} / \mathrm{c})^{2}<Q^{2}<0.3(\mathrm{GeV} / \mathrm{c})^{2}$. The scaling function for all of these cases as a function of the scaling variable $y$ are very similar-with deviations from the mean less than $10 \%$ which is probably due to binding 
energy effects. Note from the caption that all of these examples were calculated with electron scattering angles of $45^{\circ}$ since, as we will show later, there is some breakdown of scaling at lower energies when the electron scattering angle is changed significantly.

In Fig. 2, we show the same results as in Fig. 1, but with electron Coulomb distortion turned on. We refer to this case as the distorted wave impulse approximation (DWIA). For heavier nuclei, electron Coulomb distortion shifts the scaling curves toward the right side. However, the deviations from scaling due to Coulomb distortions do not seem to be too large and the largest effect occurs for larger positive values of $y$. In Fig. 3, we show the same results as in Fig. 1, but with both the final state interaction and the electron Coulomb distortion included.

Clearly the final state interaction in this kinematic range leads to rather large violations of scaling which is also observed experimentally. For example, Fig. 4 shows the comparison of our theoretical results with the experimental data for ${ }^{12} \mathrm{C},{ }^{56} \mathrm{Fe}$, and ${ }^{197} \mathrm{Au}$ measured at SLAC [21]. The incident electron energy is $E=2.02 \mathrm{GeV}$ and the electron scattering angle is $\theta=15^{\circ}$. Under these kinematic conditions, the energy transfer is below the pion production peak (except for large positive values of $y$ )and hence the quasielastic peak is well separated from inelastic processes. Note that our model, see also [12, 20], describes the quasielastic process for all three nuclei quite well and furthermore, scaling is observed for the two heavier nuclei $\left({ }^{56} \mathrm{Fe}\right.$ and $\left.{ }^{197} \mathrm{Au}\right)$ when the quasielastic process dominates $(y<0.1)$. However, the lighter nucleus $\left({ }^{12} \mathrm{C}\right)$ does not scale with the heavier ones. This deviation is due to a combination of electron Coulomb distortion and final state interaction effects.

In order to improve scaling we introduce a modified scaling variable $y^{\prime}$ given by $y^{\prime}=$ $y-(N / Z)\left|E_{b}\right|_{a v}$ where $\left|E_{b}\right|_{a v}$ is the average of the absolute binding energy for all the bound nucleons. This approach is meant to remove differences among nuclei including binding energy effects as suggested in Ref. [26, 27] and may permit scaling for quasielastic scattering from different nuclei. In Fig. 5, we show the new scaling functions for ${ }^{208} \mathrm{~Pb},{ }^{197} \mathrm{Au},{ }^{56} \mathrm{Fe}$, ${ }^{40} \mathrm{Ca}$, and ${ }^{12} \mathrm{C}$ at the same kinematics as Fig. 3. Comparing Figs. 3 and 5 clearly shows that $y^{\prime}$ improves scaling to some degree although some deviations from scaling remain.

In Fig. 6, we compare the scaling function to the experimental data measured at Bates [3] on ${ }^{40} \mathrm{Ca}$ for three cases. The solid line and $\bullet$ are for incident electron energy $E=739$ $\mathrm{MeV}$ and scattering angle $\theta=45.5^{\circ}$, the dotted line and $\mathbf{\square}$ for $E=372 \mathrm{MeV}$ and $\theta=90^{\circ}$, and the dashed line and $\boldsymbol{\Delta}$ represent $E=367 \mathrm{MeV}$ and $\theta=140^{\circ}$. The scaling functions 
have the same shape and the peaks lie at the same position, but the backward angle results (dashed curve and $\boldsymbol{\Delta}$ ) have a different magnitude from the others. From these calculations, we see that scaling at these somewhat lower energies is affected by the electron scattering angle. This result is in agreement with the studies reported by Caballero et al. [32] that final state interactions affect longitudinal and transverse contributions differentially since changing the electron scattering angle changes the longitudinal and transverse contributions to the cross section. Note that these calculations agree with the experimental data relatively well as in our previous results [3, 5, 35]. In order to investigate the dependence of scaling at lower four momentum transfer values on $\theta$, we calculated y-scaling with Coulomb distortion and final state interactions for only the protons in ${ }^{208} \mathrm{~Pb}(\mathrm{Z}=82$ and $\mathrm{N}=0)$ as shown in Fig. 7 and for only neutrons ( $\mathrm{Z}=0$ and $\mathrm{N}=126$ ) as shown in Fig. 8. As expected, the scaling function for the proton only results show a much larger dependence on the scattering angle due to the changing representation of the longitudinal and transverse response. The neutron only scaling function contains much less longitudinal contributions (due to the motion of the magnetic moment) and varies less with electron scattering angle.

In Fig. 9, we investigate scaling at larger values of the four momentum transfer as a function of electron scattering angle $\theta$ for quasielastic scattering on ${ }^{208} \mathrm{~Pb}$ when both Coulomb distortion and final state interaction are included. Based on this result and other cases we have examined, scaling is only weakly dependent on $\theta$ at these higher kinematic values although there is still some deviation at very large electron scattering angles. Furthermore, as shown in Fig 10 scaling for different nuclei still works well if all cases are calculated at the same backward scattering angle. Clearly as the final state interaction decreases scaling improves. In Fig. 11, we show the new scaling functions at high incident energy, $E=1.5$ $\mathrm{GeV}$, for the scattering angle $\theta=30^{\circ}$ in terms of $y^{\prime}$ as in Fig. 5. Clearly scaling is greatly improved even at this intermediate four momentum transfer region as long as we do not vary the electron scattering angles.

In summary, we have investigated $y$-scaling of the inclusive $\left(e, e^{\prime}\right)$ reaction from $A=12$, $20,56,197$, and 208 in the quasielastic region which is kinematically isolated from inelastic scattering. We use a realistic nuclear model describes the available data quite well and our calculations include electron Coulomb distortion. As shown in Figs. 6] and 9, the scaling function $F(y)$ is not independent of electron scattering angle with particularly strong dependence at backward scattering angles for lower values of the four momentum transfer. 
However, we have demonstrated that if one restricts the variation of the electron scattering angles (thereby not changing the longitudinal transverse mix significantly), scaling still holds at these lower kinematic values. Furthermore, as the energy increases, the dependence of scaling on electron scattering angles is greatly reduced. We also found that $y$-scaling breaks down at lower kinematic values due to the final state interaction. Again, as the energy increases (and the final state interaction weakens) the breakdown of scaling is greatly reduced. We also used our model to investigate the different binding energy effects and final state interaction effects in different target nuclei. We do find some improvement in scaling by modifying the definition of the scaling variable $y$ to include binding effects. Finally, electron Coulomb distortion does disrupt scaling to some degree, but does not play a significant role.

In conclusion, we confirm that the final state interaction is the primary cause of the scaling violation in the quasielastic region for lower energies, but we find that scaling improves dramatically at larger four momentum transfer. Our results show that at larger four momentum transfer values, scaling holds across a range of nuclei quite well. Our model results, which agree very well with the available data, suggest that scaling can be used at larger four momentum transfer values to subtract out the quasielastic contributions to the $\left(e, e^{\prime}\right)$ cross section from nuclei so that inelastic contributions which contribute incoherently to the measured cross section can be investigated. Furthermore, our results suggests that as the

energies increase the scaling function obtained from different nuclei and at different electron scattering energies differ at most by only 10 to $20 \%$.

\section{Acknowledgements}

One of us (Wright) wishes to acknowledge partial support of this work from a research grant from the Department of Energy.

[1] Z. E. Meziani et al., Nucl. Phys. A446, 113 (1985); Phys. Rev. Lett. 52, 2130 (1984); 54, 1233 (1985).

[2] M. Deady et al., Phys. Rev. C 33, 1897 (1986); 28, 631 (1983); C. C. Blatchley, J. J. LeRose, O. E. Pruet, P. D. Zimmerman, C. F. Williamson, and M. Deady, Phys. Rev. C 34, 1243 (1986). 
[3] C. F. Williamson et al., Phys. Rev. C 56, 3152 (1997); T. C. Yates et al., Phys. Lett. B 312 , $382(1993)$.

[4] A. Zghiche, et al., Nucl. Phys. A573, 513 (1994); P. Guèye, et al., Phys. Rev. C 60, 044308 (1999).

[5] Yanhe Jin, D. S. Onley, and L. E. Wright, Phys. Rev. C 45, 1333 (1992).

[6] P. M. Boucher and J. W. Van Orden, Phys. Rev. C 43, 582 (1991).

[7] C.R. Chinn, A. Picklesimer and J.W. Van Orden, Phys. Rev. C 40, 790 (1989).

[8] M. Traini, S. Turck-Chiéze, and A. Zghiche, Phys. Rev. C 38, 2799 (1988); Phys. Lett. B 213, 1 (1988).

[9] T. W. Donnelly, J. W. Van Orden, T. de Forest, Jr., and W. C. Hermans, Phys. Lett. B 76, 393 (1978).

[10] K. S. Kim, L. E. Wright, Yanhe Jin, and D. W. Kosik, Phys. Rev. C 54, 2515(1996).

[11] K. S. Kim, L. E. Wright, and D. A. Resler, Phys. Rev. C 64, 044607 (2001).

[12] K. S. Kim and L. E. Wright, Phys. Rev. C 67, 054604 (2003).

[13] Yanhe Jin, D. S. Onley, and L. E. Wright, Phys. Rev. C 45, 1311 (1992); 50, 168 (1994).

[14] Yanhe Jin, J.K. Zhang, D.S. Onley and L.E. Wright, Phys. Rev. C 47, 2024 (1993).

[15] J. M. Udias, P. Sarriguren, E. Moya de Guerra, E. Garrido, and J. A. Caballero, Phys. Rev. C48, 2731 (1993).

[16] K. S. Kim and L. E. Wright, Phys. Rev. C 56, 302 (1997); 60, 067604 (1999).

[17] C. J. Horowitz and B. D. Serot, Nucl. Phys. A368, 503 (1981).

[18] E. D. Cooper, S. Hama, B. Clark, and R. L. Mercer, Phys. Rev. C 47, 297 (1993).

[19] Hungchong Kim, C. J. Horowitz, and M. R. Frank, Phys. Rev. C 51, 792 (1995).

[20] K. S. Kim and L. E. Wright, Phys. Rev. C 68, 027601 (2003).

[21] D. B. Day et al., Phys. Rev. Lett. 59, 427 (1987).

[22] G. B. West, Phys. Rep. 18, 263 (1975).

[23] D. Day et al., Phys. Rev. C 40, 1011 (1989); 48, 1849 (1993).

[24] E. Pace and G. Salmé, Phys. Lett. 110B, 411 (1982).

[25] C. Ciofi degli Atti, Nuovo Cimento A 76, 330 (1983).

[26] C. Ciofi degli Atti, E. Pace, and G. Salmé, Phys. Rev. C 39, 259 (1989).

[27] C. Ciofi degli Atti, E. Pace, and G. Salmé, Phys. Rev. C 43, 1155 (1991).

[28] D. B. Day, J. S. McCarthy, T. W. Donnelly, and I. Sick, Annu. Rev. Nucl. Part. Sci. 40, 357 
(1990).

[29] J.E. Amaro, M.B. Barbaro, J.A. Caballero, T.W. Donnelly, and J.M. Udias, Phys. Rev. C75, 034613 (2007).

[30] J. Arrington et al., Phys. Rev. Lett. 82, 2056 (1999); Phys. Rev. C 64, 014602 (2001); J. Arrington, Ph.D. Thesis, Cal. Tech, 1998. URL: http://www.krl.caltech.edu/ johna/thesis.

[31] A. N. Antonov, M. K. Gaidarov, D. N. Kadrev, E. Moya de Guerra, and J. M. Udias, Phys. Rev. C69, 044321 (2004); A. N. Antonov, M. V. Ivanov M. K. Gaidarov, E. Moya de Guerra, P. Sarriguren, and J. M. Udias, Phys. Rev. C73, 047302 (2006); A. N. Antonov, M. V. Ivanov, M. K. Gaidarov, E. Moya de Guerra, J. A. Caballero, M. B. Barbaro, J. M. Udias, and P. Sarrigen, Phys. Rev. C74, 054603 (2006).

[32] J. A. Caballero, J. E. Amaro, M. B. Barbaro, T. W. Donnelly, and J. M. Udias, arXiv:0705.1429v1 [nucl-th], 10 May 2007.

[33] T. De Forest Jr., Nucl. Phys. A392, 232 (1983).

[34] E. Pace and G. Salmé, Phys. Lett. 110B, 411 (1982).

[35] K. S. Kim and Myung Ki Cheoun, Phys. Rev. C 67, 034603 (2003). 


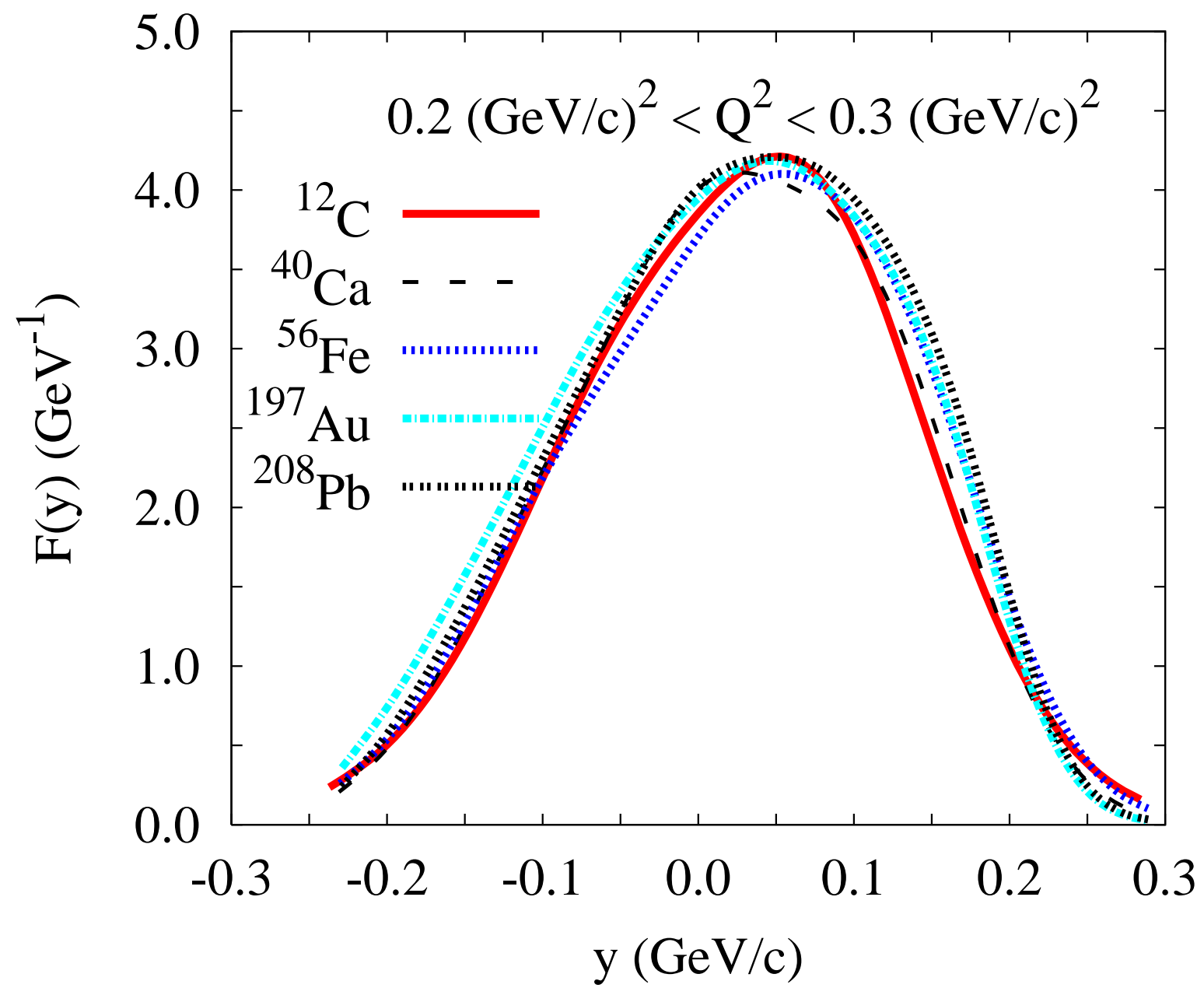

FIG. 1: The $y$-scaling functions for ${ }^{12} \mathrm{C},{ }^{40} \mathrm{Ca},{ }^{56} \mathrm{Fe},{ }^{197} \mathrm{Au}$, and ${ }^{208} \mathrm{~Pb}$ with incident electron energy $750 \mathrm{MeV}$ and scattering angle $45^{\circ}$. The calculations do not include the final state interaction of the outgoing nucleons nor electron Coulomb distortion at the four momentum squared of approximately $0.2(\mathrm{GeV} / \mathrm{c})^{2}<Q^{2}<0.3(\mathrm{GeV} / \mathrm{c})^{2}$. 


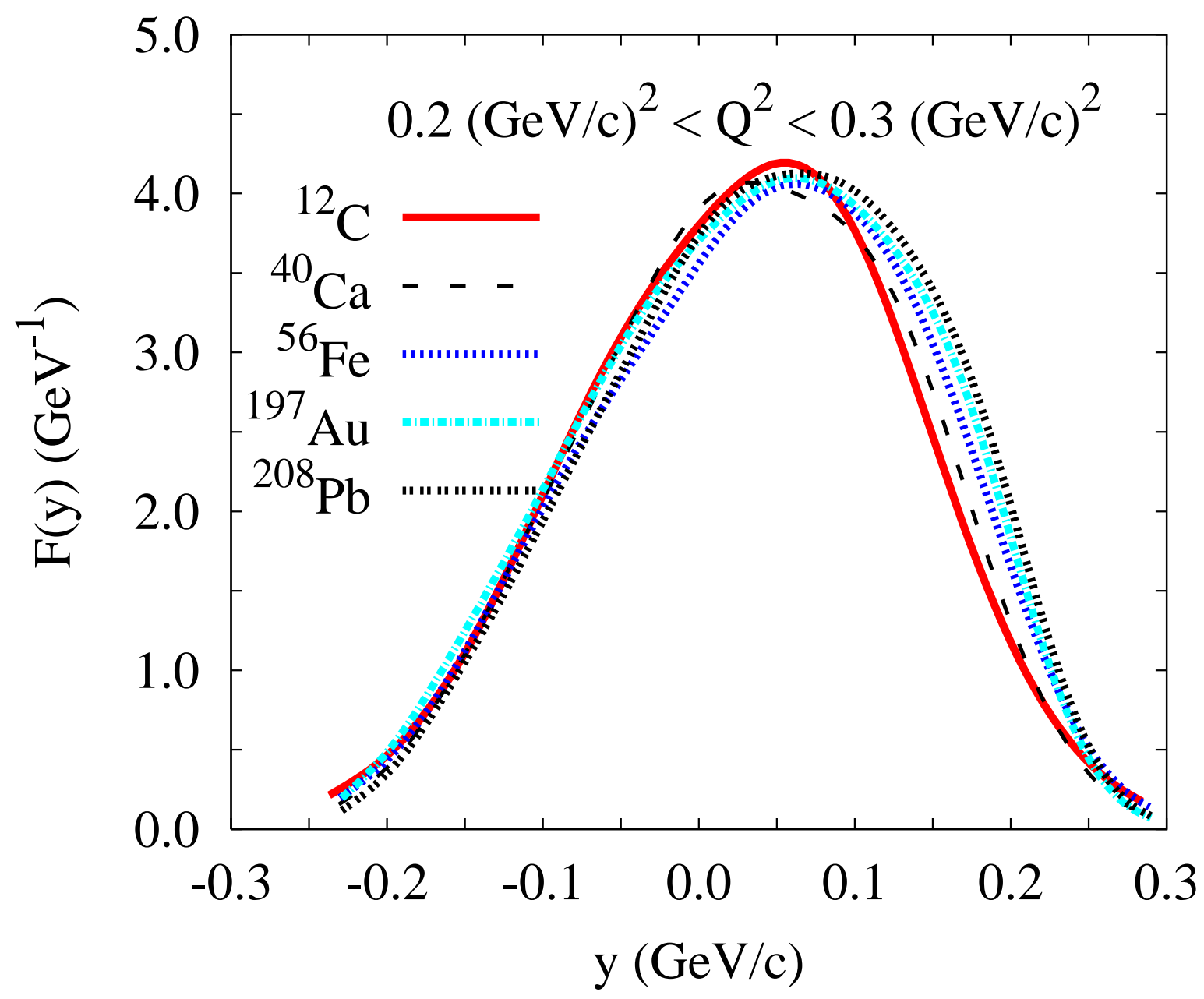

FIG. 2: The same as in Fig. 1 except electron Coulomb distortion is included.. 


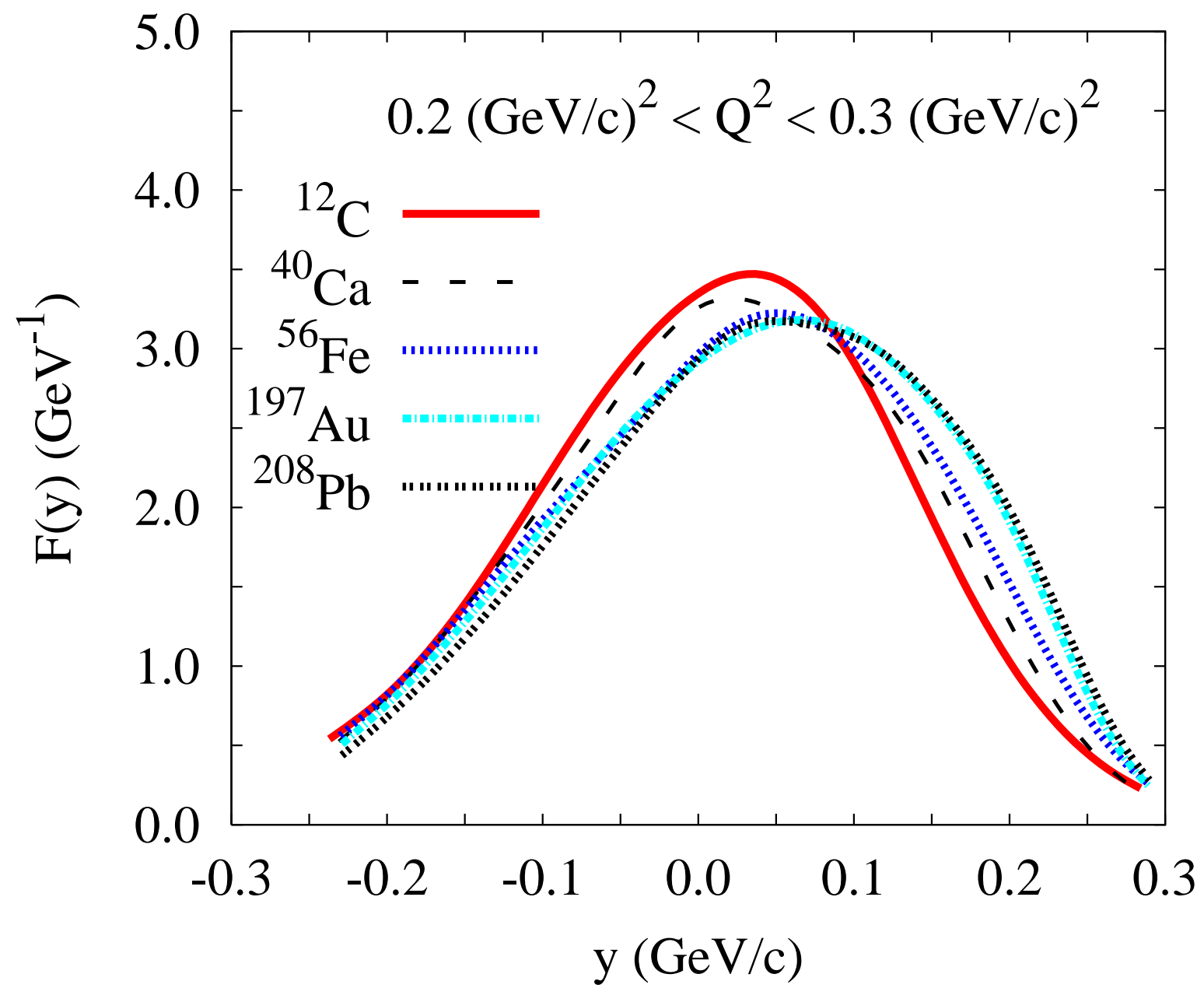

FIG. 3: The same as in Fig. 11 except both electron Coulomb distortion and the final state interaction of the outgoing nucleons are included. 


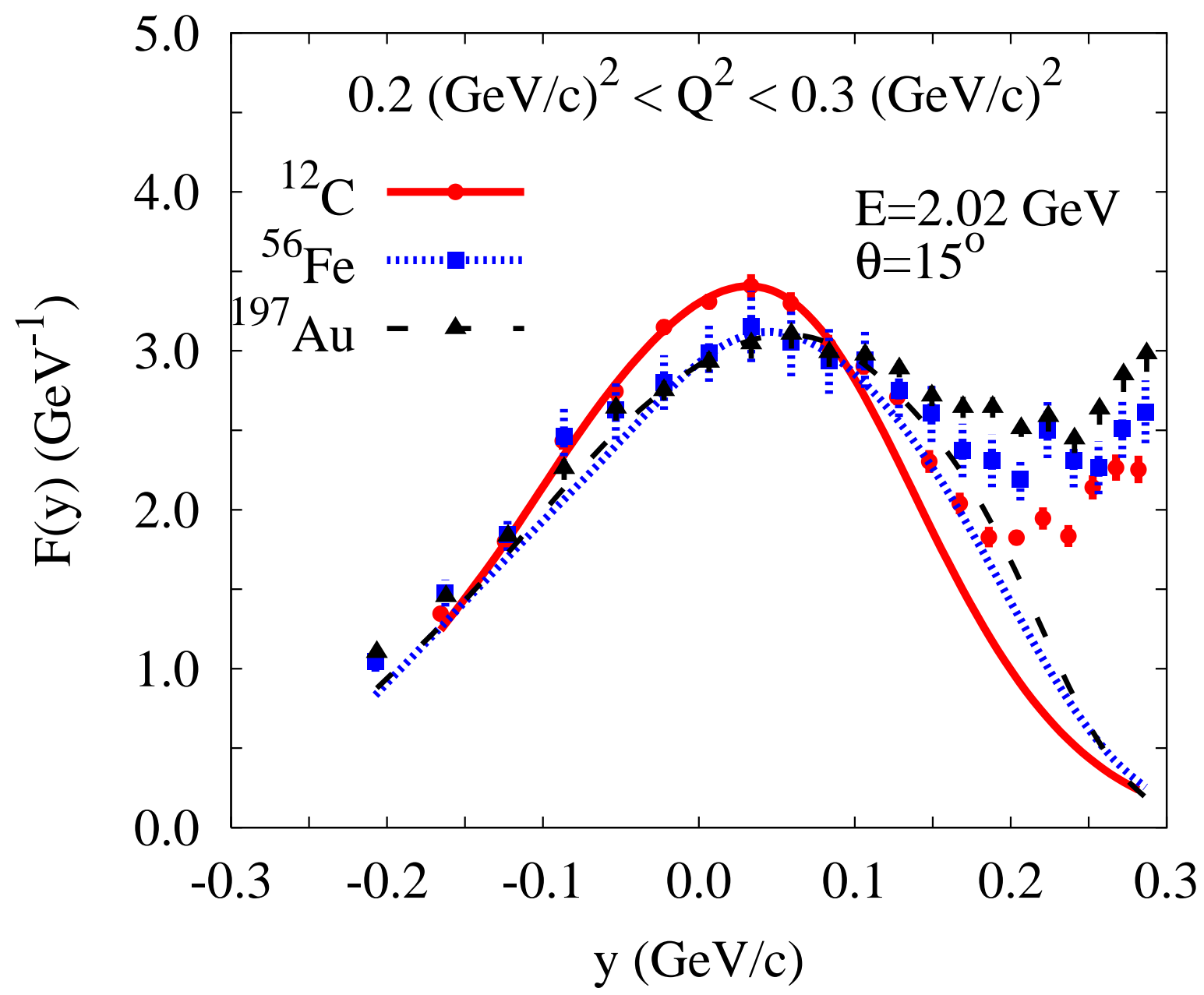

FIG. 4: The $y$-scaling functions for three different target nuclei from ${ }^{12} \mathrm{C}$ (sold and $\bullet$ ), ${ }^{56} \mathrm{Fe}$ (dot and $\mathbf{\square}$ ), and ${ }^{197} \mathrm{Au}$ (dash and $\boldsymbol{\Delta}$ ). The electron incident energy is $E=2.02 \mathrm{GeV}$ and the scattering angle is $\theta=15^{\circ}$. The experimental data are from SLAC [21]. 


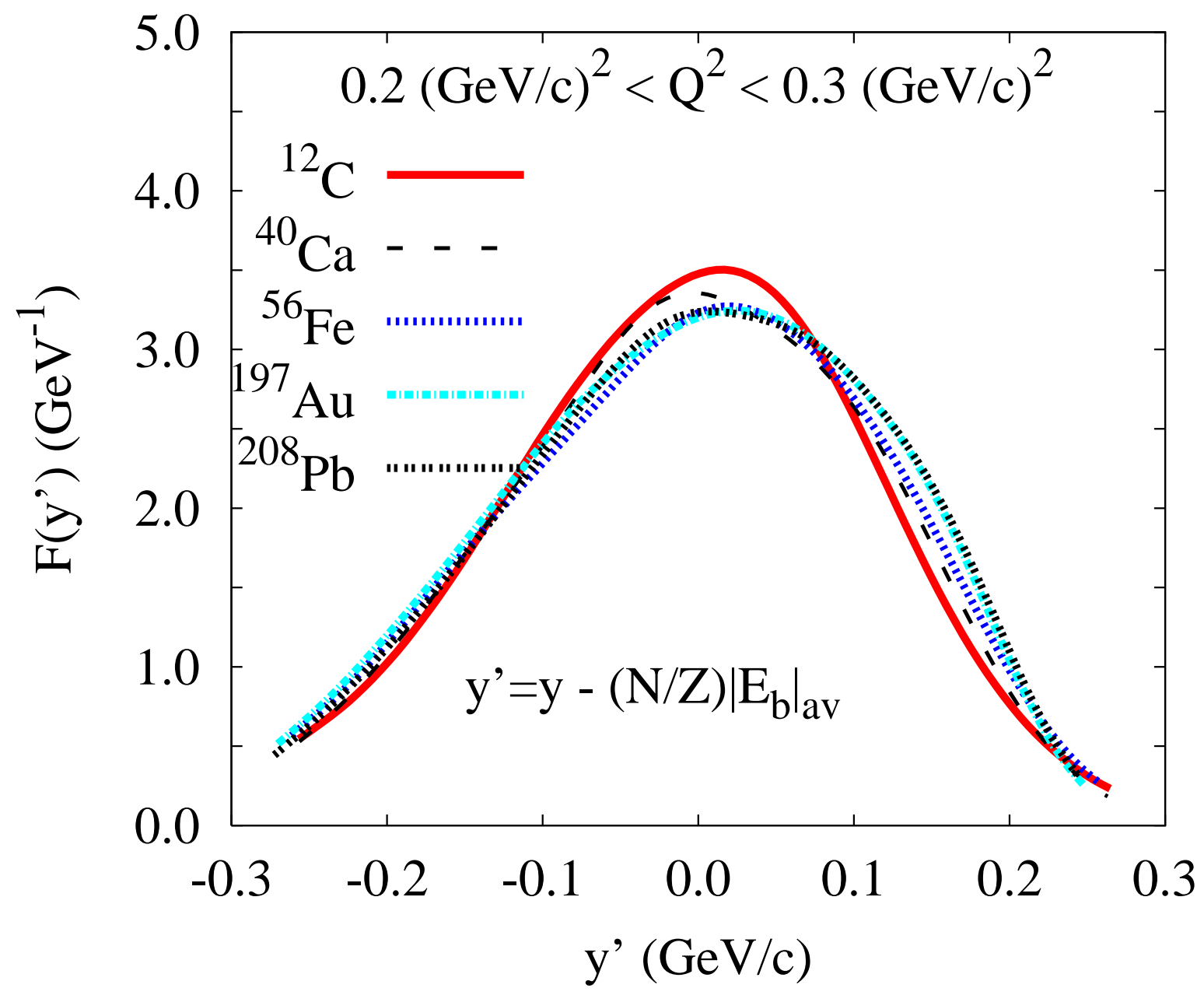

FIG. 5: The same kinematics as in Fig. 3 except plotted as a function of the new $y^{\prime}$-scaling variable. 


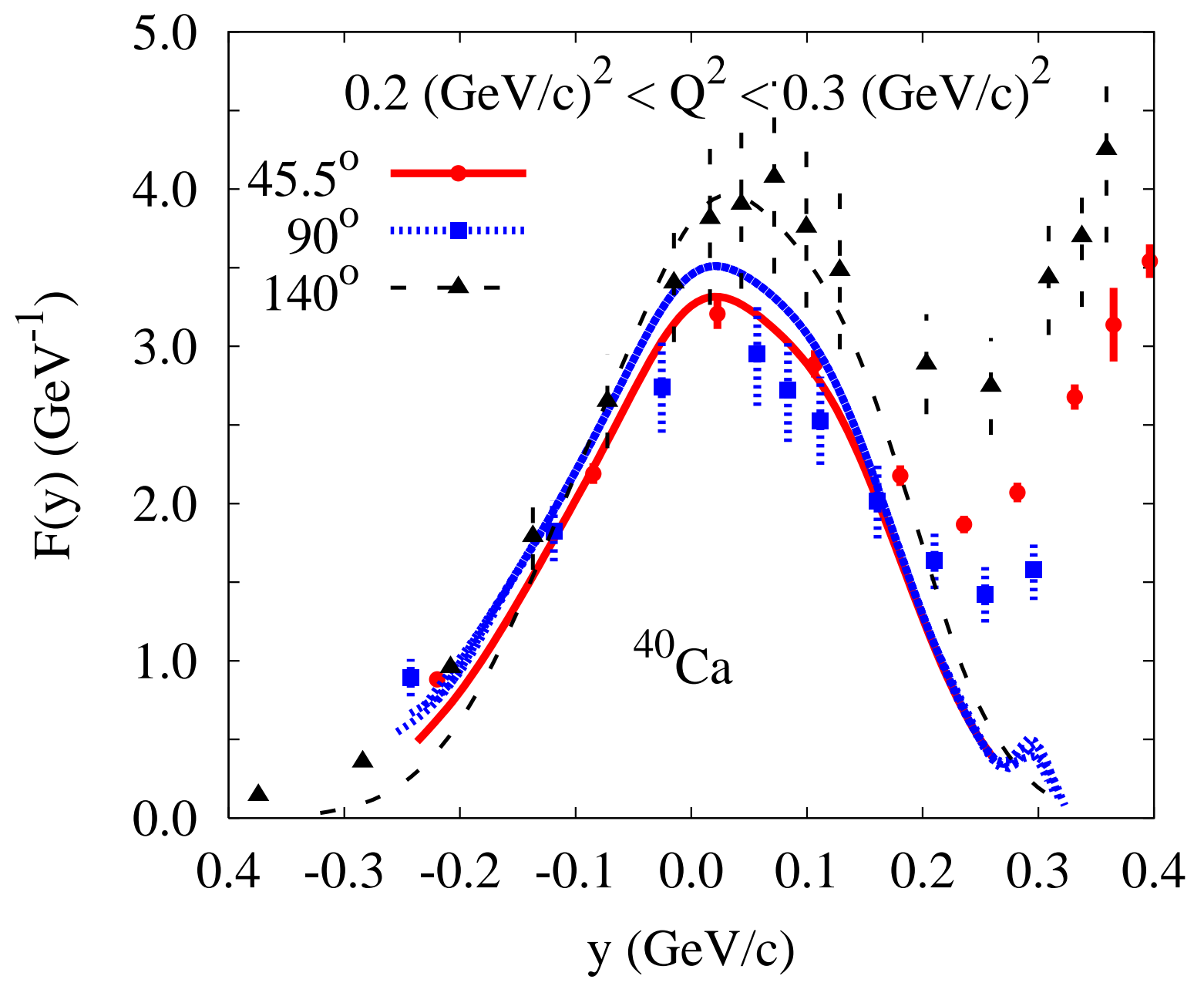

FIG. 6: The $y$-scaling functions for ${ }^{40} \mathrm{Ca}$ at three different electron energies, $E=739 \mathrm{MeV}$ and scattering angle $\theta=45.5^{\circ}$ (solid and $\bullet$ ), $E=375 \mathrm{MeV}$ and $\theta=90^{\circ}$ (dot and $\mathbf{\square}$ ), and $E=367$ $\mathrm{MeV}$ and $\theta=140^{\circ}$ (dash and $\boldsymbol{\Delta}$ ). The experimental data are from Bates [3] . 


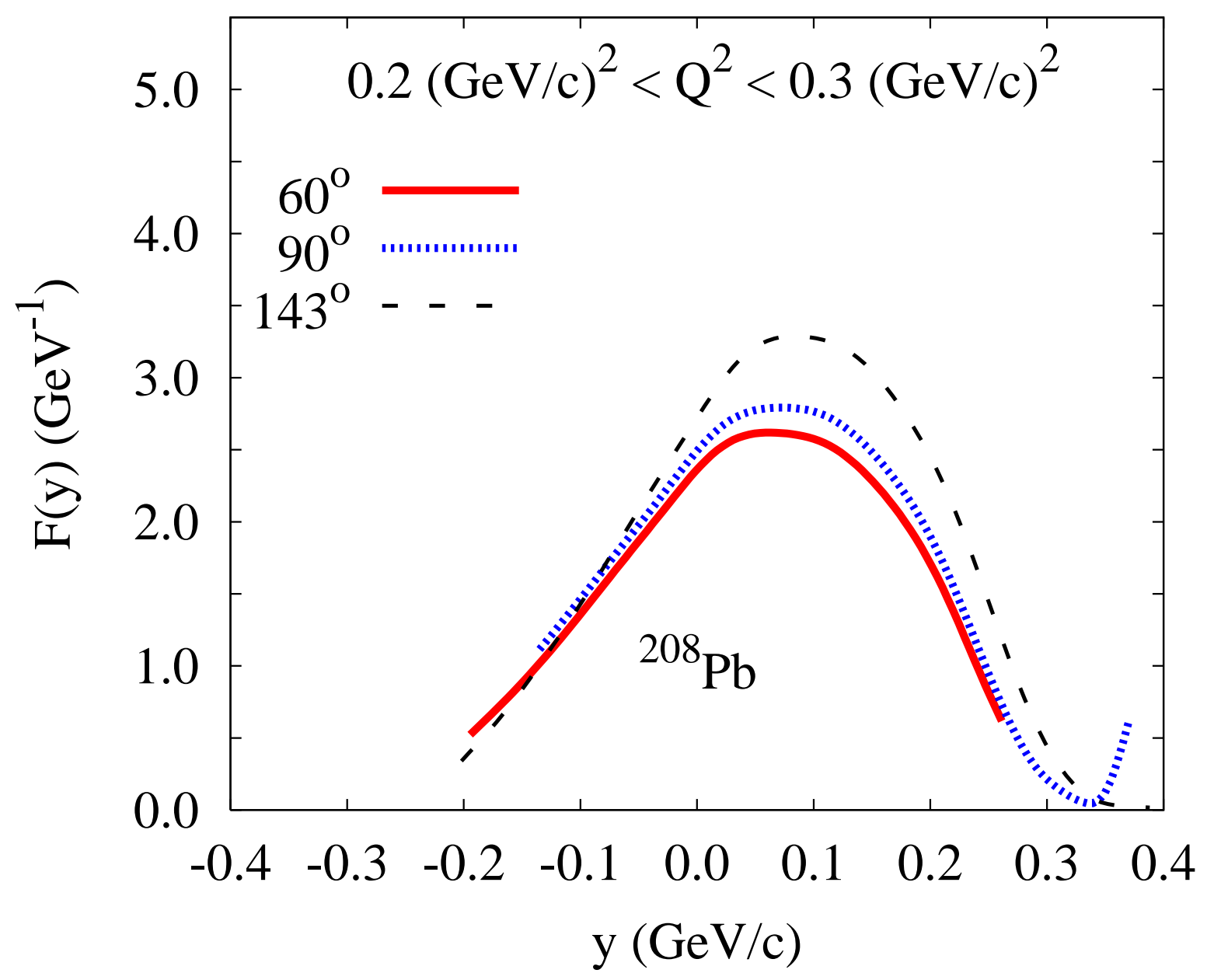

FIG. 7: The $y$-scaling functions for only the protons from ${ }^{208} \mathrm{~Pb}$ at three different electron energies, $E=550 \mathrm{MeV}$ and scattering angle $\theta=60^{\circ}, E=354 \mathrm{MeV}$ and $\theta=90^{\circ}$, and $E=310 \mathrm{MeV}$ and $\theta=143^{\circ}$ with Coulomb distortion and final state interaction included. 


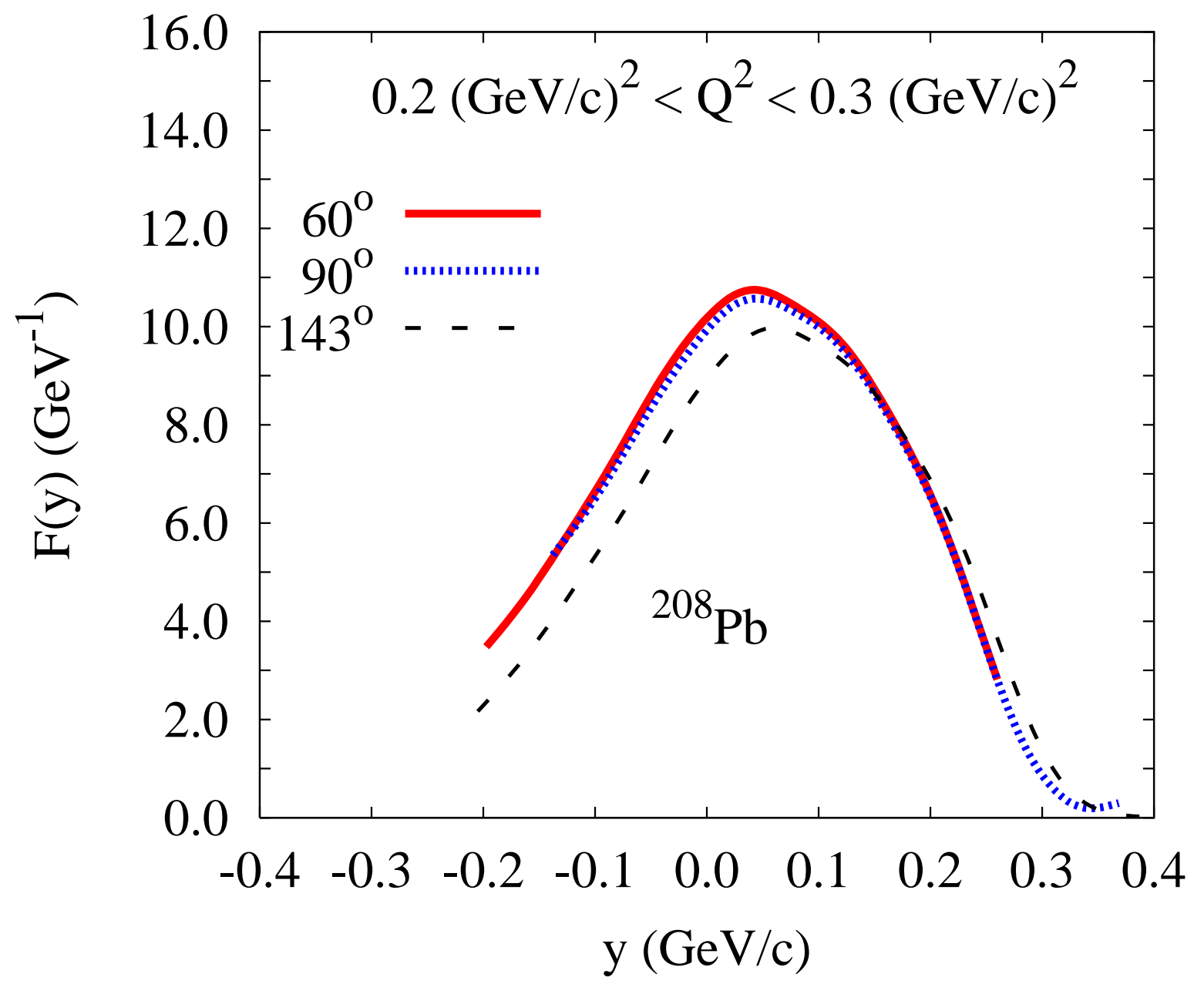

FIG. 8: The same as in Fig. 7 except for neutrons only. 


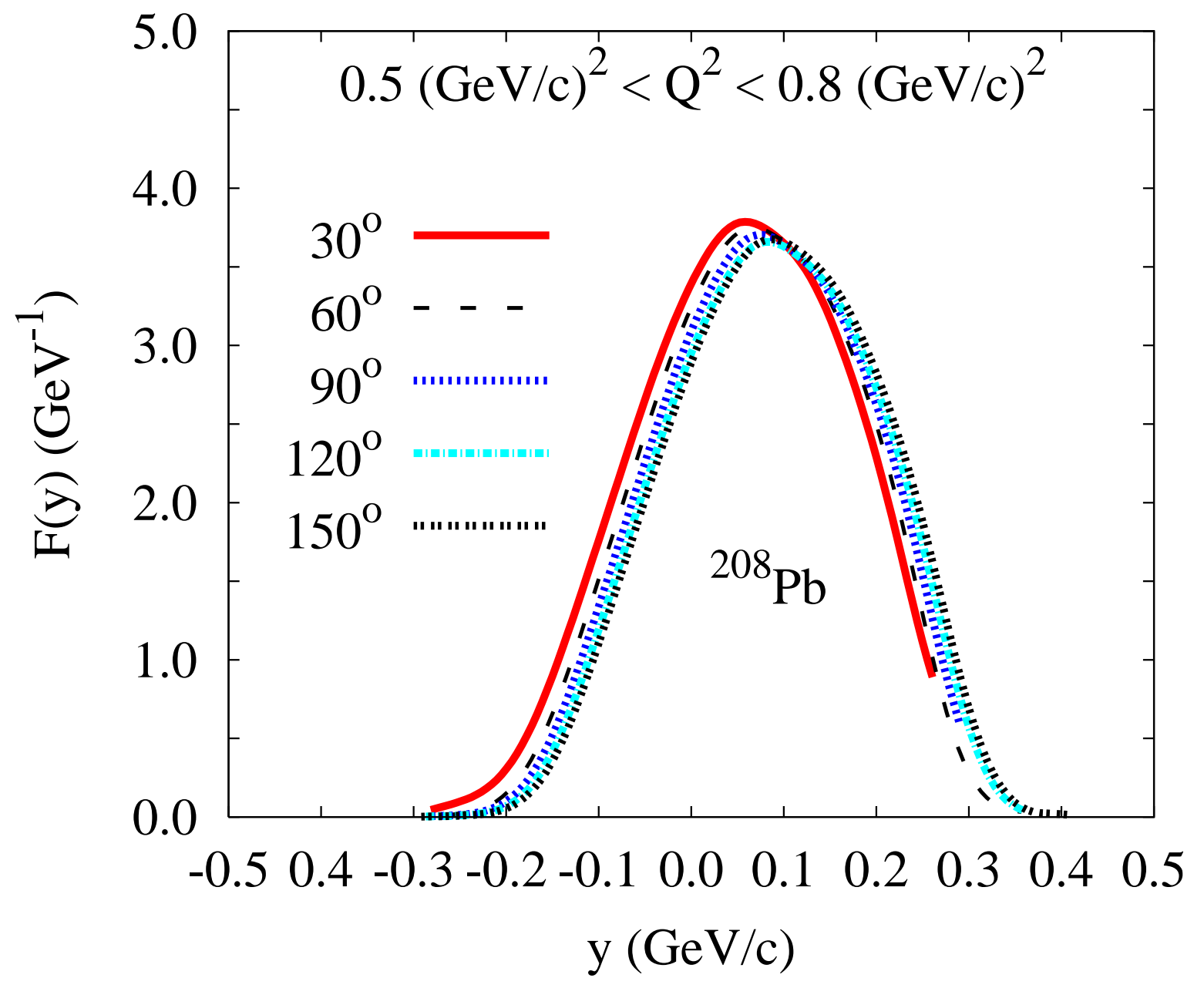

FIG. 9: The $y$-scaling functions for ${ }^{208} \mathrm{~Pb}$ both with final state interaction and Coulomb distortion at five different electron kinematics, $E=1830 \mathrm{MeV}$ and scattering angle $\theta=30^{\circ}$ (solid and red), $1000 \mathrm{MeV}$ and $60^{\circ}$ (dash and black), $740 \mathrm{MeV}$ and $90^{\circ}$ (dot and blue), $630 \mathrm{MeV}$ and $120^{\circ}$ (dashdot and skyblue), and $580 \mathrm{MeV}$ and $150^{\circ}$ (two-dot and black). The range of the four momentum squared is approximately $0.5(\mathrm{GeV} / \mathrm{c})^{2}<Q^{2}<0.8(\mathrm{GeV} / \mathrm{c})^{2}$. 


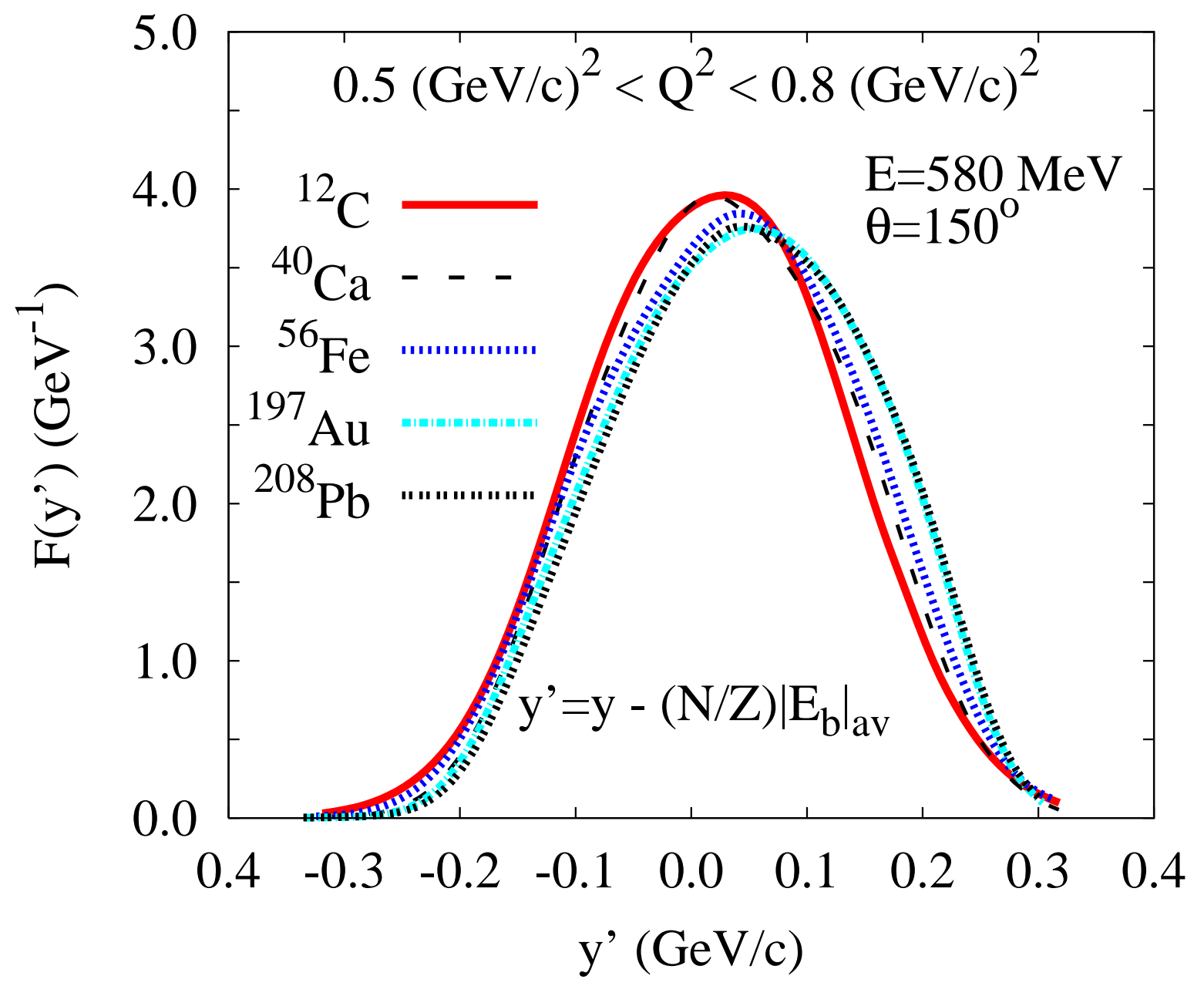

FIG. 10: The $y^{\prime}$-scaling functions for several nuclei with final state interaction and Coulomb distortion at the incident electron energy $E=580 \mathrm{MeV}$ and scattering angle $\theta=150^{\circ}$. The range of the four momentum squared is approximately $0.5(\mathrm{GeV} / \mathrm{c})^{2}<Q^{2}<0.8(\mathrm{GeV} / \mathrm{c})^{2}$. 


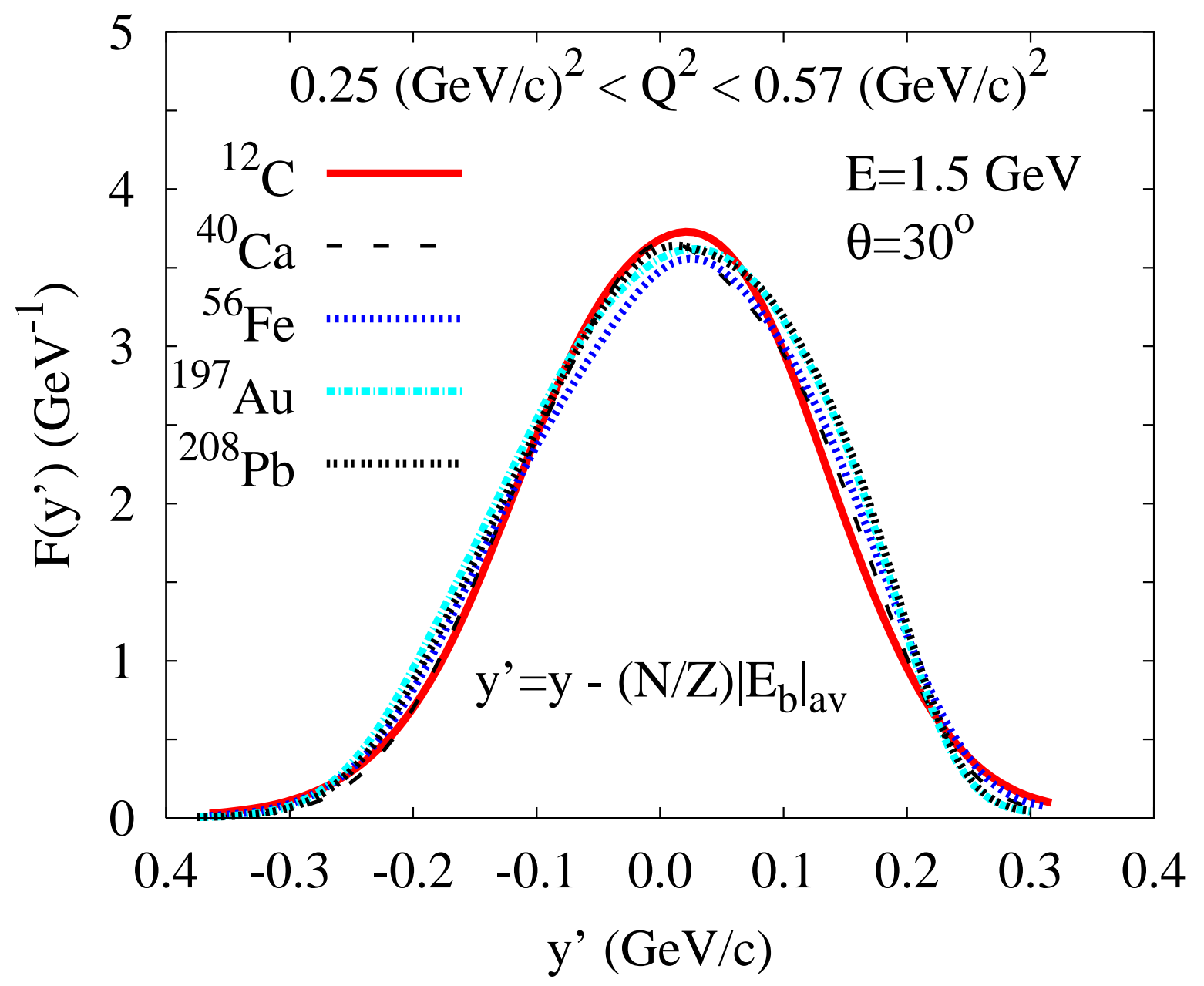

FIG. 11: The new $y^{\prime}$-scaling functions for the high electron energy $E=1.5 \mathrm{GeV}$ and scattering angle $\theta=30^{\circ}$ from several nuclei. 